\section{Audiology and Neurotology}

Yuri Agrawal, Baltimore, MD, USA Ola Alqudah, Riyadh, Saudi Arabia Susan Arndt, Freiburg, Germany Atılım Atılgan, Istanbul, Turkey Paul Avan, Auvergne, France Daniel H. Baker, York, UK Manohar Bance, Cambridge, UK Maurizio Barbara, Rome, Italy Matthew Robert Bartindale, Joliet, USA François Bergeron, Québec, QC, Canada Daniele Bernardeschi, Paris, France Eldre Beukes, Cambridge, UK Philip Bird, Christchurch, New Zealand Bojana Bukurov, Belgrade, Serbia Matthew Bush, Lexington, KY, USA Barbara Canlon, Stockholm, Sweden Nathan Cass, Nashville, TN, USA Renjie Chai, Nanjing, China Alexander Chern, New York, NY, USA Thomas M. Coate, Washington, DC, USA

Raanan Cohen-Kerem, Haifa, Israel Abeir Dabbous, Cairo, Egypt Valerie Dahm, Vienna, Austria Vincent Darrouzet, Bordeaux, France Marije de Jong, Utrecht, The Netherlands Raquel De Sousa Lobo Ferreira Querido, New York, NY, USA Sumitrajit Dhar, Evanston, IL, USA Peter Dixon, Toronto, ON, Canada Hamid R. Djalilian, Irvine, CA, USA Ward R. Drennan, Seattle, WA, USA Raffeale Dubbioso, Naples, Italy Fakih Cihat Eravci, Konya, Turkey Isaac Erbele, Fort Sam Houston, TX, USA Adrien Eshraghi, Miami, FL, USA Juan Carlos Falcon Gonzalez, Las Palmas de Gran Canaria, Spain Carolyn Falls, Toronto, ON, Canada John Ferraro, Kansas City, KS, USA Douglas Fitzpatrick, Chapel Hill, NC, USA

David Friedmann, New York, NY, USA

Takwa A. Gabr, Kafrelsheikh, Egypt

Bruce Gantz, Iowa City, IA, USA

Imre Gerlinger, Pécs, Hungary
Rachel Elizabeth Glade, Fayetteville, AR, USA

Rudolf Glückert, Innsbruck, Austria

Justin S. Golub, New York, NY, USA

Karen A. Gordon, Toronto, ON, Canada

Madhuri Gore, Bangalore, India

Tim Green, London, UK

John Greinwald, Cincinnati, OH, USA

Maeher Grewal, New York, NY, USA

Samuel Gubbels, Denver, CO, USA

Stavros Hatzopoulos, Ferrara, Italy

Ohad Hilly, Petack Tikwa, Israel

Michael Hoa, Bethesda, MD, USA

Derek Hoare, Nottingham, UK

Jourdan T. Holder, Nashville, TN, USA

Clemens Honeder, Vienna, Austria

Ulrich Hoppe, Erlangen, Germany

Arata Horii, Niigata, Japan

Lihui Huang, Beijing, China

Alexander Huber, Zurich, Switzerland

Alexandria Irace, New York, NY, USA

Yulian Jin, Yanbian, China

Figen Karabekiroğlu, Tokat, Turkey

Hannah Keppler, Ghent, Belgium

Edward C. Killan, Leeds, UK

H. Jeffrey Kim, Washington, DC, USA

Sung Huhn Kim, Seoul, Republic of Korea

Bla Koritnik, Ljubljana, Slovenia

Ebru Kosemihal, Nikosia, Cyprus

Takaomi Kurioka, Tokorozawa, Japan

Jeong-Sug Kyong, Seoul, Korea

Emilie Lacroix, Louvain, Belgium

Ho Yun Lee, Seoul, Republic of Korea

Hyo-Jeong Lee, Anyang-si,

Republic of Korea

Eugenijus Lesinskas, Vilnius, Lithuania

Tien-Chen Liu, Hsinchu City, Taiwan

Ivan Axel Lopez, Los Angeles, CA, USA

Jose Antonio Lopez-Escamez, Granada, Spain

Enrique A. Lopez-Poveda, Salamanca, Spain

Jose Faibes Lubianca Neto, Porto Alegre, Brazil

Rui-Xia Ma, Ningxia, China

Alexis Maffert, Brest, France
Lennart Magnusson, Gothenburg, Sweden Patrizia Mancini, Rome, Italy

Manuel Manrique, Pamplona, Spain

Diane Masser-Frye, San Diego, CA, USA

Ryan McCreery, Boys Town, NE, USA

Franck Michel, Aarhus, Denmark

Paul Mick, Saskatoon, SK, Canada

Philipp Mittmann, Berlin, Germany

Aaron C. Moberly, Columbus, OH, USA

Samer Mohsen, Damascus, Syria

David Moore, Cincinnati, OH, USA

Isabelle Mosnier, Paris, France

Sarah Mowry, Cleveland, OH, USA

Togay Müderris, Izmir, Turkey

Banu Mujdeci, Ankara, Turkey

Kevin Munro, Manchester, UK

R. Nash, London, UK

Jack H. Noble, Nashville, TN, USA

Brendan O'Connell, Chapel Hill, NC, USA

Kevin K. Ohlemiller, St. Louis, MO, USA

Hidehiko Okamoto, Otawara, Japan

Tadao Okayasu, Kashihara, Japan

Yahav Oron, Tel Aviv, Israel

Juan C. Ospina, Bogota, Colombia

Ryohei Oya, Osaka, Japan

Kourosh Parham, Farmington, CT, USA

Kathy Pichora Fuller, Toronto, ON, Canada

Davide Pisani, Catanzaro, Italy

Cache Pitt, Logan, UT, USA

Prashanth Prabhu, Mysuru, India

Julie Purdy, San Diego, CA, USA

Nicola Quaranta, Bari, Italy

Kristen Rak, Würzburg, Germany

Massimo Ralli, Rome, Italy

Christin Ray, Columbus, OH, USA

Aaron Remenschneider, Boston, MA, USA

Ellen Rhoades, Washington, DC, USA

Dominik Riss, Vienna, Austria

Christof Röösli, Zurich, Switzerland

Lukas Rüttiger, Tübingen, Germany

Shaza Mahmoud Saleh, Riyadh,

Saudi Arabia

Ravi Samy, Cincinnati, OH, USA 
Hiroaki Sato, Iwate, Japan

Alexander Saxby, Sydney, NSW, Australia

Reinhold Schatzer, Innsbruck, Austria

Kara Schvartz-Leyzac, Charleston, SC,

USA

Young Joon Seo, Wonju, Republic of Korea

Scott Shapiro, Hoboken, NJ, USA

Xiaorui Shi, Eugene, OR, USA

Niraj Kumar Singh, Mysore, India

Heidi K. Slager, Ann Arbor, MI, USA

Ad F.M. Snik, Nijmegen, The Netherlands

Samuel Söderqvist, Helsinki, Finland

Jae-Jin Song, Seoul, Korea

Nathaniel Spencer, Washington, DC, USA

Georg Mathias Sprinzl, St. Pölten, Austria

Nirmal Srinivasan, Towson, MD, USA

Olivier Sterkers, Paris, France

Shawn Stevens, Phoenix, AZ, USA
Josh Sturm, New York, NY, USA

Qing Sun, Beijing, China

Maja Svrakic, Long Island, NY, USA

Joyce Tang, Singapore, Singapore

George Tavartkiladze, Moscow,

Russian Federation

Somia Tawfik, Cairo, Egypt

Viral Tejani, Iowa City, IA, USA

Linda Thibodeau, Dallas, TX, USA

Hans G.X.M. Thomeer, Utrecht,

The Netherlands

Ingo Todt, Bielefeld, Germany

Hiroaki Tsukano, Chapel Hill, NC, USA

Richard Tyler, Iowa City, IA, USA

Marc M. van Wanrooij, Nijmegen,

The Netherlands

Anita Vereb Vereb, Ann Arbor, MI, USA

Nicolas Verhaert, Leuven, Belgium
Christophe Vincent, Lille, France

P. Ashley Wackym, New Brunswick, NJ, USA

Susan B. Waltzman, New York, NY, USA

Bryan K. Ward, Baltimore, MD, USA

Matthew B. Winn, Minneapolis, MN, USA

Chen-Chi Wu, Taipei, Taiwan

Shu Hui Xu, Singapore, Singapore

Yi-Ho Young, Taipei, Taiwan

Olaf Zagólski, Kraków, Poland

Thomas Zahnert, Dresden, Germany

Andrzej Zarowski, Antwerpen, Belgium

Qing Zhang, Xian, China

Jing Zheng, Chicago, IL, USA

Hao Zhu, Kansas City, MO, USA

Stefan Zirn, Offenburg, Germany

Teresa Zwolan, Ann Arbor, MI, USA

Terry A. Zwolan, Ann Arbor, MI, USA 